\title{
Vortex Dynamics and Dissipation under High-Amplitude Microwave Drive
}

\author{
Mattia Checchin $\circledast^{*}$ and A. Grassellino \\ Fermi National Accelerator Laboratory, Batavia, Illinois 60510, USA
}

(Received 13 April 2020; revised 4 September 2020; accepted 8 September 2020; published 13 October 2020)

\begin{abstract}
In this paper, we describe the vortex dynamics under a high-amplitude microwave drive and its effect on the surface resistance of superconductors. The vortex surface resistance is calculated with a Monte Carlo approach, where the vortex equation of motion is solved for a collection of vortex flux lines, each oscillating within a random pinning landscape. This approach is capable of providing a detailed description of the microscopic vortex dynamics and in turn important insights into the microwave-field-amplitude dependence of the vortex surface resistance. The numerical simulations are compared against experimental data of vortex surface resistance at high microwave amplitude measured by means of bulk niobium superconducting radio-frequency cavities operating at $1.3 \mathrm{GHz}$. The good qualitative agreement of the simulations and experiments suggests that the nonlinear dependence of the trapped-flux surface resistance with the microwave field amplitude is generated by progressive microwave depinning and vortex jumps.
\end{abstract}

DOI: 10.1103/PhysRevApplied.14.044018

\section{INTRODUCTION}

Upon cool down below critical temperature in the presence of an external magnetic field, magnetic flux quanta - so-called vortices - can exist in thermodynamic equilibrium in the mixed state of type-II superconductors $[1,2]$.

Below the lower critical field, vortices are not stable in the superconductor; however, because of the occurrence of defects in real materials, vortices get pinned and survive even in the Meissner state. An incomplete Meissner effect, where $100 \%$ of the applied field is trapped by the superconductor, can be achieved with superconducting radio-frequency (SRF) cavities by field cooling slowly across the transition, as experimentally observed by many studies [3-6].

Driven by radio-frequency (rf) and microwave fields, vortices oscillate the dissipating power and contribute to the total surface resistance. Other than vortex oscillation, other contributions to the surface resistance are the temperature-dependent surface resistance due to thermally excited quasiparticles above the gap [7], surface resistance due to subgap states [8], proximity-coupled normalconducting inclusions (or oxides) [9], nanohydrides [10],

*checchin@fnal.gov

Published by the American Physical Society under the terms of the Creative Commons Attribution 4.0 International license. Further distribution of this work must maintain attribution to the author(s) and the published article's title, journal citation, and DOI. and dielectric dissipation due to spurious two-level systems at low microwave amplitudes and low temperatures $[11,12]$.Vortex dissipation under a microwave drive is a topic that has been under investigation for many decades. Initial studies on type-II superconducting films [13] brought about the development of models to describe the vortex-surface-resistance dependence on the frequency $[14,15]$. Since then, several other studies have investigated the microwave behavior of conventional superconductors [16-19], cuprates [20-22], and iron-based superconductors [23-25] in the presence of vortices. In parallel, many theoretical works allow us to describe the expected penetration depth change due to vortex oscillation [26], the vortex-surface-impedance-temperature dependence in high- $T_{c}$ superconductors [27], and the precise electrodynamics of vortex lattices in the mixed state [28].

In the framework of modern particle accelerators employing SRF cavities as accelerating devices, dissipation due to vortex motion under a microwave drive is a topic of central importance, inasmuch as trapped fields in the order of approximately $10 \mathrm{mG}$ can increase the surface resistance of about $1 \mathrm{n} \Omega$ [6], a large value for state-of-theart cavities capable of achieving surface-resistance values as low as $4 \mathrm{n} \Omega$ at $2 \mathrm{~K}[29,30]$.

Early studies on $S$-band SRF bulk Nb cavities[31] recognized the effect of vortices on the surface resistance, identifying the microwave-amplitude dependence of the latter to follow a nonlinear behavior. Later studies have shown that up to moderate gradients, the microwave-fieldamplitude dependence of the vortex surface resistance in $\mathrm{Nb}$ sputtered on copper SRF cavities is linear [32]. Studies of vortex dynamics in cavity-grade $\mathrm{Nb}$ and a theoretical description of the phenomenon observed in the 
zero microwave-field-amplitude approximation have also been studied in detail [33].

More recently, intensive studies of the trapped-flux surface resistance at low and moderate accelerating gradients as a function of the electron mean free path $(l)$ have discovered a bell-like trend as a function of $l[6,34]$, which is described well by the interplay of pinning- and fluxflow-limited dissipation [35-37]. These studies have also reported an almost linear dependence of the vortex dissipation with the microwave field amplitude in the resonator up to moderate values.

The microwave-field-amplitude dependence can be understood as the occurrence of nonquadratic terms in the pinning potential [38]. The linear dependence has been shown to be well described by adding a cubic term to a parabolic pinning potential in the vortex equation of motion [38]. However, this approach is limited, since it is built around the single-vortex dynamics, while in the material there are several vortices contributing to the overall dissipation, each one interacting with its surroundings.

A second approach [39], which overcomes the latter issue, implements a mean-field method to solve the vortex equation of motion for collective weak pinning. In this case, the microwave-field-amplitude dependence has two regimes: (i) hysteretic losses at low amplitudes, where the dependence is linear; and (ii) viscous losses at higher amplitudes, where the surface resistance saturates to a constant value.

In the present work, the high-amplitude microwave field dependence of the vortex surface resistance is explored by comparing the experimental data acquired for a highperforming single-cell $\mathrm{Nb}$ accelerating cavity to the surface resistance calculated by means of numerical simulations. The model developed is based on a Monte Carlo approach that assumes a random distribution of the pinning potentials in the vortex-oscillation plane. The simulation scheme presented here allows us to gather insights into the dynamics of vortex oscillations in random pinning potentials such as vortex jumps and microwave depinning, which are not captured by previous models but are key to correct interpretation of the experimental data.

\section{EXPERIMENTAL DATA}

The experimental data of the vortex surface resistance is calculated from microwave measurements of a singlecell TESLA-type [40] bulk niobium cavity operating at $1.3 \mathrm{GHz}$.

The cavity surface is prepared with a series of treatments designed to increase the maximum accelerating gradient achievable by the resonator, in order to capture the power-dissipation fingerprint due to the high-amplitude microwave behavior of vortices. The cavity is initially electropolished to remove $200 \mu \mathrm{m}$ and subsequently baked at $800{ }^{\circ} \mathrm{C}$ for $3 \mathrm{~h}$ to degas hydrogen and release internal stress. After this initial surface preparation, the cavity is rinsed using high-pressure water in a class 10 clean room to remove field-emitting particles from the inner surface and assembled with the antennas needed for microwave characterization. The cavity is then evacuated and baked at $120^{\circ} \mathrm{C}$ for $48 \mathrm{~h}$ in situ.

The resonator is equipped with three single-axis flux-gate magnetometers (from Bartington Instruments), located symmetrically around the cavity equator, a set of Helmholtz coils aligned coaxially to the cavity beam pipe, and a temperature-mapping system (Tmap) [41] to precisely monitor the cavity temperature rise during the measurements.

The cavity is initially zero-field cooled (ZFC) and the $Q$ factor versus the accelerating gradient data is acquired at $1.5 \mathrm{~K}$. During this measurement, the resonator reaches a maximum peak magnetic field $\left(B_{p}\right)$ of $204.5 \mathrm{mT}$ $(48 \mathrm{MV} / \mathrm{m})$ with a $Q$ factor of $9.6 \times 10^{9}$. The same cavity is then field cooled (FC) very slowly to $1.5 \mathrm{~K}$ four times, trapping, respectively, $7.5 \mathrm{mG}, 30 \mathrm{mG}, 50 \mathrm{mG}$, and $100 \mathrm{mG}$ upon the superconducting transition.

As described in detail in Ref. [6], the vortex surface resistance is calculated by subtracting the ZFC data from the $\mathrm{FC}$ data acquired at the same temperature. By performing the measurement at $1.5 \mathrm{~K}$, the thermal contribution to the surface resistance is negligible and the systematic error introduced by the accuracy of the temperature control in the dewar is minimized.

In Fig. 1(a), the vortex surface resistance $\left(R_{\mathrm{fl}}\right)$ is reported as a function of the peak magnetic field. The normalized vortex surface resistance over the magnetic field trapped during the cool down $\left(B_{0}\right)$ - the so-called trapped-flux sensitivity - is reported in Fig. 1(b). As shown in Fig. 1(b), once the vortex surface resistance is normalized by $B_{0}$, the field dependence appears the same within the measurement error (approximately $10 \%$ of the $R_{\mathrm{fl}}$ value) $[6,42]$. This means that, within the range of $B_{0}$ and $B_{p}$ investigated, the peak magnetic-field-amplitude dependence of $R_{\mathrm{fl}}$ is dictated by the vortex dynamics and not by thermal feedback. If thermal feedback was playing a role, then the slope of $R_{\mathrm{fl}} / B_{0}$ would be steeper for higher $B_{0}$, since our experimental approach to extract $R_{\mathrm{fl}}$ cannot discriminate thermal-feedback effects.

In agreement with previous measurement of the vortex surface resistance as a function of the accelerating gradient, performed in thin-film and bulk $\mathrm{Nb}$ cavities [6,32], $R_{\mathrm{fl}}$ increases almost linearly up to moderate microwave fields (approximately $80 \mathrm{mT}$ ). For higher field amplitudes, the vortex surface resistance deviates from the almost linear growth and the slope increases with $B_{p}$, leading to higher values of resistance.

Experimental data are also acquired by means of the Tmap system, which is composed of 36 boards arranged symmetrically around the cavity, each incorporating 15 

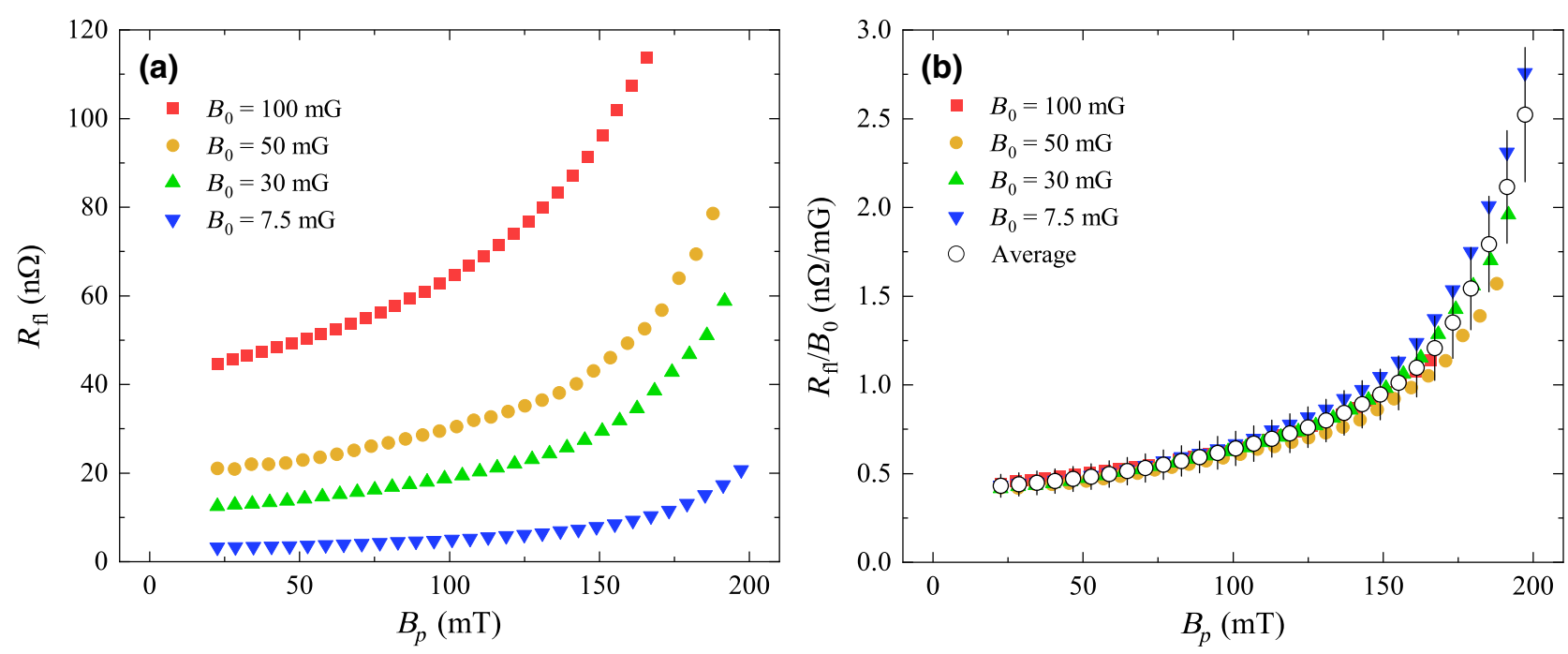

FIG. 1. (a) The experimental data of the trapped-flux surface resistance up to high values of the peak magnetic field. (b) The normalized $R_{\mathrm{fl}}$ over the trapped magnetic field.

thermometers in contact with the outer surface of the cavity, as sketched in Fig. 2(a).

The data are acquired at $B_{p}=166 \mathrm{mT}$ both in the ZFC experiment and in the FC experiment, with $100 \mathrm{mG}$ applied during the cool down. The ZFC Tmap data are then subtracted from the FC data in order to obtain the heating pattern due to trapped vortices only. The temperaturemapping data are shown in Fig. 2(c). The board number is reported as the abscissa, while the thermometer numbers are given as the ordinate.

Interestingly, the vortex dissipation shows a specific pattern with heating, concentrated symmetrically with respect to the equator of the cavity (the equator location corresponds to thermometer number 8 ), whereas, instead, the temperature differential $\Delta T_{\text {out }}$ is lower than in neighboring areas and tends to zero.

In this configuration, the trapped magnetic field $B_{0}$ is parallel to the cavity beam axis [as shown in Fig. 2(a)] and at the equator the microwave currents are parallel to the trapped field. Because of the angular dependence of the Lorentz force that drives the vortex oscillation, this geometrical effect leads to lower dissipation at the equator compared to areas of the cavity where the flux has an angle almost perpendicular to the microwave currents.

It is important to point out that this behavior is intertwined with the modulation of the microwave currents, which varies with the local amplitude of the surface magnetic field $B$ at the cavity surface. $B$ is almost constant from thermometer 4 to thermometer 12 - the surface peak field $B_{p}$ is at thermometers 6 and 10 , and it decays rapidly to zero from thermometer 3 to 1 and from 13 to 15 [as shown in Fig. 2(b)].

This modulation of the surface field $B$ explains why the vortex dissipation is almost zero at the cavity iris (thermometers 1 and 15), where the flux and currents are, instead, close to orthogonality. (a)

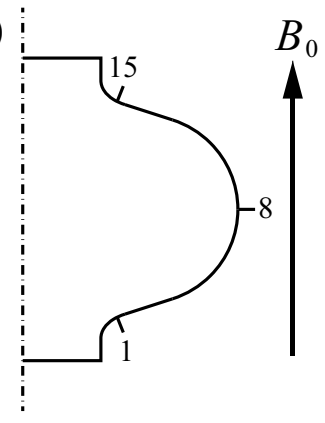

(b)

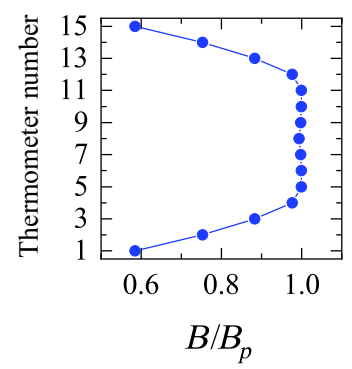

(c)

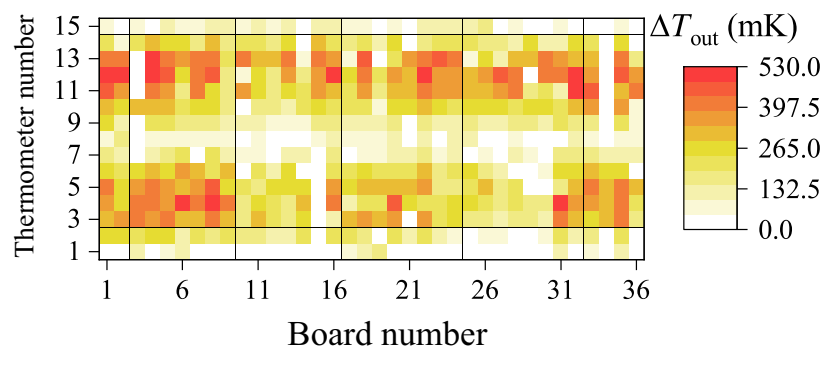

FIG. 2. (a) A sketch of the cavity section, indicating the locations of the thermometers. (b) The ratio between the local magnetic field at the surface over the peak magnetic field at each thermometer location. (c) The Tmap, showing the vortex dissipation collected at $B_{p}=166 \mathrm{mT}$. 


\section{VORTEX DYNAMICS}

Numerical simulations of vortex dynamics are performed in order to shed light on the field dependence of the vortex surface resistance.

We assume a superconducting surface at $z=0$ parallel to the $x-y$ plane, where the semispace $z>0$ is filled by superconducting niobium and the semispace $z<0$ is vacuum free space. The magnetic field trapped $\left(B_{0}\right)$ is parallel to the $z$ axis and we assume that upon transition the magnetic flux is subdivided into individual vortices that do not interact with each other, $B_{0} \ll B_{c 2}$.

The microwave excitation-with frequency $f=\omega /$ $2 \pi$ - at the superconducting surface has a magnetic field oscillating along the $x$ axis, inducing a current parallel to the $y$ axis, which decays exponentially in the material along $z$, with a characteristic decay length equal to the penetration depth $\lambda$ [43]. The amplitude of the microwave field coincides with the peak magnetic field $B_{p}$.

Neglecting the inertial term and assuming in a first approximation that the penetration depth $\lambda$ does not depend on the vortex motion, since we are considering sparse trapped vortices when $B_{0}<B_{c 1}$ [26], the vortex equation of motion has the form

$$
\eta \dot{u}(t, z)=\epsilon u^{\prime \prime}(t, z)+f_{p}(t, z)+\gamma B_{p} \cos (\omega t) e^{-z / \lambda},
$$

where $u(t, z)$ is the vortex displacement as a function of the time and depth $z, \dot{u}$ is the time partial derivative, $u^{\prime \prime}$ is the second partial derivative along $z$, and $\gamma=\phi_{0} /\left(\mu_{0} \lambda\right)$. $\epsilon$ is the single-vortex line tension [44], defined as

$$
\epsilon=\frac{\phi_{0}^{2}}{4 \pi \mu_{0} \lambda^{2}}\left[\frac{1}{2}+\ln \kappa+e^{-0.4-0.8 \ln \kappa-0.1 \ln ^{2} \kappa}\right],
$$

with $\kappa=\lambda / \xi$ [45]. The vortex viscosity $\eta$ is equal to $\sigma \phi_{0} B_{c 2}$ [46] and $\sigma$ is the niobium normal-state conductivity at low temperature. The pinning force per unit length is introduced through $f_{p}(t, z)$.

The numerical simulations are performed for finite values of the electron mean free path $(l)$. The penetration depth and coherence length are calculated as described by Tinkham [47]. For isotropic superconductors such as niobium, the spatial Fourier transform of Pippard's kernel [48] $K(q, l, T)$ is defined as

$$
\begin{aligned}
K(q, l, T)= & \frac{\xi(l, T)}{\xi_{0}(T) \lambda_{L}(T)^{2}}\left\{\frac{3}{2(q \xi(l, T))^{3}}\right. \\
& \left.+\left[\left(1+q^{2} \xi(l, T)^{2}\right) \tan ^{-1} q \xi(l, T)-q \xi(l, T)\right]\right\},
\end{aligned}
$$

where $\lambda_{L}(T)$ is approximated by the two-fluid model definition $\lambda_{L}(T)=\lambda_{L}(0)\left[1-\left(T / T_{c}\right)^{4}\right]^{-1 / 2}[49]$ and the $T$ dependence of $\xi$ and $\xi_{0}$ is derived from BCS theory [50]:

$$
\begin{aligned}
\xi(l, T) & =\left[\frac{J(0, T)}{\xi_{0}(0)}+\frac{1}{l}\right]^{-1}, \\
\xi_{0}(T) & =\frac{\xi_{0}(0)}{J(0, T)}
\end{aligned}
$$

Here, $J(0, T)$ is the real-space kernel as defined in Ref. [50,51]:

$$
J(0, T)=\left[\frac{\lambda_{L}(T)}{\lambda_{L}(0)}\right]^{2} \frac{\Delta(T)}{\Delta(0)} \tanh \frac{\Delta(T)}{2 \kappa_{B} T},
$$

where $\Delta(T) / \Delta(0)=\left\{\cos \left[\pi / 2\left(T / T_{c}\right)^{2}\right]\right\}^{1 / 2}[52]$ and $\Delta(0)$ is the energy gap at $T=0 \mathrm{~K}$.

The penetration depth $\lambda(l, T)$ is then calculated numerically for specular reflection at the boundary as follows:

$$
\lambda(l, T)=\frac{2}{\pi} \int_{0}^{\infty} \frac{d q}{K(q, l, T)+q^{2}} .
$$

The parameters $\lambda_{L}(0)$ and $\xi_{0}(0)$ represent the London penetration depth and the coherence length of niobium in the clean limit at $T=0$ respectively equal to $39 \mathrm{~nm}$ and $38 \mathrm{~nm}$ [53].

\section{A. Pinning landscape}

Vortex pinning in superconductors is explained as the tendency of the system to minimize the total loss in condensation energy. Being a vortex itself, a singularity in the condensation energy, it interacts with areas in the material where superconductivity is suppressed or absent in order to minimize the overall condensation-energy loss.

This mechanism intrinsically implies the minimum dimension for a defect to be an efficient pinning center as the coherence length $\xi$, which represents the minimum length over which a given change in the condensed electron density can be made [54].

The coherence length in niobium is of the order of $38 \mathrm{~nm}$ or lower when scattering centers are present as described above. This implies that any defect in the material with a dimension of the order of $\xi$ or larger could pin vortices. Due to their small transverse dimension (approximately $1-5 \mathrm{~nm}$ ), grain boundaries and single dislocations cannot be considered efficient pinning centers, whereas dislocation tangles and material nonuniformity can extend for as much as hundreds of nanometers, allowing for more efficient pinning $[55,56]$.

In niobium, normal-conducting $\mathrm{Nb}_{x} \mathrm{H}_{y}$ precipitates might also behave as pinning centers. Hydrogen is always present as an interstitial in $\mathrm{Nb}$, with a concentration that depends on the thermal history of the material. Depending on the hydrogen concentration, $\mathrm{Nb}_{x} \mathrm{H}_{y}$ precipitates can 
have dimensions that range from a couple of nanometers to microns. The cavity under study is prepared in such a way that it minimizes the number and dimension of $\mathrm{Nb}$ hydrides by performing thermal treatments at $800^{\circ} \mathrm{C}$ and $120^{\circ} \mathrm{C}$ [57]. The dimension of the $\mathrm{Nb}_{x} \mathrm{H}_{y}$ precipitates is expected to be of the order of $10 \mathrm{~nm}$ or smaller [10]; hence, in this instance, we expect that $\mathrm{Nb}$ hydrides are less effective pinning centers than dislocation tangles and we disregard their contribution.

Due to the defective nature of real materials, many defects may simultaneously pin a single vortex along its length, making it virtually impossible to measure the pinning potential directly, especially for bulk materials. Theoretical calculations [58] suggest that isolated pinning centers can be approximated by Lorentzian functions of the type $U(r)=-U_{0} /\left[1+(r / \xi)^{2}\right]$, where $r$ is the radial distance from the vortex on the plane orthogonal to $\phi_{0} \hat{n}$, while experimental measurements on thin films [59] have shown that real pinning potentials can be approximated by a summation of several of these Lorentzian functions.

Similarly to what done by Labusch [60], the pinning force is calculated as $f_{p}(t, z)=-\nabla U(u(t, z), z)$, where the pinning landscape $U(x, z)$ is constructed as the summation of $N$ individual pinning potentials represented by Lorentzian functions - defined above - in the $x-z$ oscillation plane of the vortices:

$$
U(x, z)=-\sum_{i}^{N}\left[\frac{U_{i}(b \xi)^{2}}{(b \xi)^{2}+\left(x-X_{i}\right)^{2}+a_{i}\left(z-Z_{i}\right)^{2}}\right] .
$$

Here, $U_{i}, X_{i}$, and $Z_{i}$ represent, respectively, the $i$ th pinning potential energy and the $(x, z)$ coordinates in the vortex-oscillation plane, the variable $a_{i}$ is a dimensionless parameter that accounts for the anisotropy of the pinning potential along the $z$ direction, and $b$ is a multiplicative factor to account for the pinning-center dimension.

The anisotropy parameter $a_{i}$ is introduced in order to emulate elongated pinning centers such as dislocation tangles occurring along the direction of the vortex flux. As described above, dislocation tangles are expected to be the most energetically favorable pinning sites in niobium, since their transverse dimension is of the order of $\xi$ $[55,56,61]$.

The number of pinning centers $N$ is kept constant at 50 , while the coordinates $\left(X_{i}, Z_{i}\right)$ and the anisotropy values $a_{i}$ are generated at random within $-1<X_{i}<1 \mu \mathrm{m}$, $0<Z_{i}<5 \mu \mathrm{m}$, and $0.001<a_{i}<1$. The pinning-energy values $U_{i}$ are generated at random following a normal distribution centered around an average value $\left(U_{\text {ave }}\right)$ and a normal deviation $\sigma_{U}=5 \% U_{\text {ave }}$. $U_{\text {ave }}$ is defined as the pinning energy that translates to a maximum pinning force per unit length $\left(f_{p \text {,ave }}\right)$ comparable to values found in the literature. Usually, for niobium, the pinning force per unit length varies depending on the material's impurity content and the crystallographic structure in the range $10^{-6}-10^{-4}$ $\mathrm{N} / \mathrm{m}[56,62,63]$.

\section{B. Numerical simulations}

Numerical simulations of the vortex motion are defined within the $x-z$ plane domain where the pinning-force landscape is defined by means of Eq. (7), assuming distributions for the pinning energy, location, and anisotropy as described above.

The initial condition and the boundary conditions used in the calculation are as follows:

$$
\begin{aligned}
u(0, z) & =0, \\
u^{\prime}(t, 0) & =0, \\
u^{\prime}\left(t, z_{\max }\right) & =0,
\end{aligned}
$$

where $z_{\max }$ represents the maximum depth of the solution domain defined from 0 - the microwave surface - to $z_{\max }$.

In several works $[33,39]$, the boundary condition at the far edge of the solution domain is set to be $u\left(t, z_{\max }\right)=0$, implying a vortex strongly pinned in the bulk. On the contrary, in this work vortices are allowed to find their own equilibrium positions for any randomly generated pinning landscape and we set $u^{\prime}\left(t, z_{\max }\right)=0$. As we see later, this boundary-condition choice is mandatory to allow the vortex line displacement in the bulk to evolve with time until an equilibrium position is found-a condition reached after several oscillations, when the simulation converges to a steady-state solution.To perform the numerical simulations, Eqs. (1) and (8) are expressed with lengths in units of $\lambda$ and times in units of $1 / f: u=\tilde{u} \lambda, z=\tilde{z} \lambda$, $X_{i}=\tilde{X}_{i} \lambda, Z_{i}=\tilde{X}_{i} \lambda$, and $t=\tilde{t} / f$. The equation of motion then becomes

$$
\begin{aligned}
\eta \lambda f \dot{\tilde{u}}(\tilde{t}, \tilde{z})= & \frac{\epsilon}{\lambda} \tilde{u}^{\prime \prime}(\tilde{t}, \tilde{z})+f_{p}(\tilde{t}, \tilde{z}) \\
& +\gamma B_{p} \cos (2 \pi \tilde{t}) e^{-\tilde{z}}
\end{aligned}
$$

The equation of motion in Eq. (9) is solved numerically by means of the method of lines. The spatial derivatives along $z$ are discretized into a grid of $M$ points using the finite-element method, while the time variable is kept continuous. Equation (9) is thus approximated by a system of $M$ differential equations, each describing the time evolution of $\tilde{u}$ evaluated on one of the $M$ grid points. To solve Eq. (9), $M$ is chosen between 1000 and 10000 depending on the complexity of the pinning landscape.

The calculation is allowed to evolve for several periods of oscillation, until the solution converges to a steadystate condition, identified by a close trajectory in the vortex-motion phase portrait, $\dot{\tilde{u}}$ versus $\tilde{u}$. 
In Fig. 3(a), an example of the vortex-motion phase portrait at the surface $(z=0)$ calculated for surface peak field $B_{p}=50 \mathrm{mT}, f_{p \text {,ave }}=50 \mu \mathrm{N} / \mathrm{m}$ and $l=300 \mathrm{~nm}$ is shown. The motion starts with the vortex in the initial condition $[\tilde{u}(0, \tilde{z})=0]$ and evolves with time until the steady-state condition is reached.

In Fig. 3(b), the time evolution of the vortex displacement within the pinning landscape is shown. The vortex initiates its motion from the initial condition represented
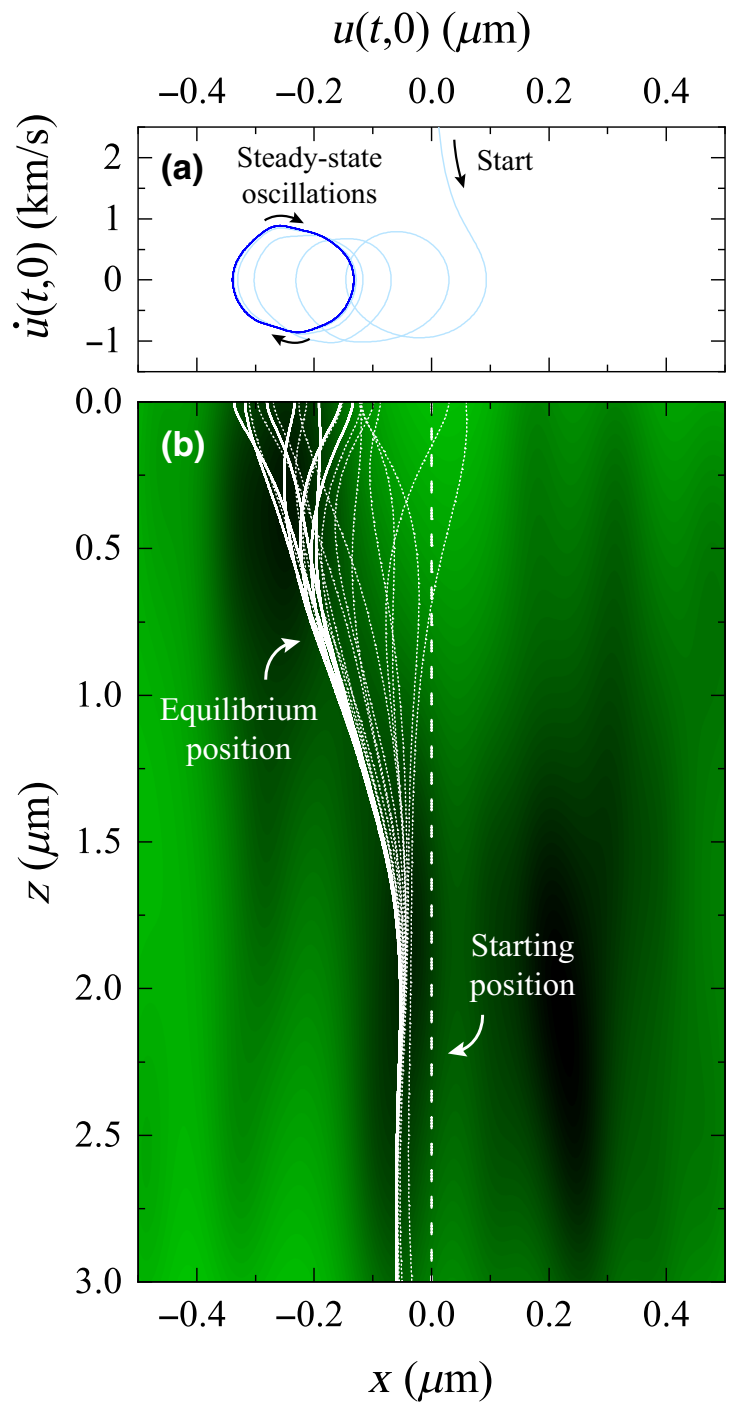

FIG. 3. An example of vortex-dynamics simulation. (a) The phase portrait of the vortex tip. The arrows indicate the direction of the vortex motion. The transition from the initial condition to the steady state is shown in light blue. (b) The time evolution of the vortex displacement with respect to its initial position is shown by the dashed line. The solid lines show the vortex motion in the steady state, while the dotted lines show the time evolution from initial condition to steady state. The pinning landscape in the background is calculated by means of Eq. (7), stronger pinning areas being shown with a darker contrast. by the straight dashed line at $x=0$. Then, it starts oscillating at the surface while moving toward negative $x$ values, attracted by a nearby pinning center (the darker region). Because of this, the initial motion of the vortex is chaotic - as shown by the phase portrait in Fig. 3(a) and by the dotted lines in Fig. 3(b) - and it eventually converges to steady-state oscillations when the vortex finds its equilibrium position attracted by a pinning center, as shown by the solid lines in Fig. 3(b) on the left-hand side of the solution domain.

As briefly described above, the vortex self-stabilization to equilibrium according to the pinning landscape is allowed by the boundary condition chosen at the far edge of the solution domain. The condition $\tilde{u}^{\prime}\left(\tilde{t}, \tilde{z}_{\max }\right)=$ 0 allows the vortex to move and reach an equilibrium position without being constrained to a specific location.

Simulations are also performed preparing the initial condition by letting the vortex relax to equilibrium before applying the driving force. The employment of such a simulation strategy results in an equivalent outcome within the simulation accuracy as if $\tilde{u}(0, \tilde{z})=0$ is used, but requires a longer time to converge to a steady-state solution. Therefore, all the simulations presented here are performed using the initial condition reported in Eq. (8).

\section{VORTEX SURFACE RESISTANCE}

During a FC experiment, we assume that all the magnetic flux applied during cool down gets trapped in the material and divided into $N_{v}$ vortices. Once an electromagnetic field is applied to the material, inducing currents parallel to the surface, the vortices oscillate, absorbing part of the electromagnetic energy dissipating power.

The power per unit of area dissipated by vortex motion is defined as

$$
\frac{d P}{d \Sigma}=\frac{1}{2 \mu_{0}^{2}} R_{\mathrm{fl}} B_{p}^{2}
$$

If we consider a situation in which the magnetic field $B_{p}$ and $R_{\mathrm{fl}}$ are constant over the surface, $R_{\mathrm{fl}}$ can be defined as

$$
R_{\mathrm{fl}}=\frac{2 \mu_{0}^{2}}{B_{p}^{2} \Sigma} P
$$

where $P$ represents the total power dissipated by the collection of the $N_{v}$ oscillating vortices.Because of the random nature of the pinning landscape in real materials, we assume that each vortex oscillates within its local pinning landscape, which differs from vortex to vortex except for its average pinning force. Under this assumption, the power $P$ represents the sum of the single-vortex dissipated powers $P_{i}^{(1)}$ calculated over the total number of $N_{v}$ 
vortices:

$$
\begin{aligned}
P & =\sum_{i}^{N_{v}} P_{i}^{(1)} \\
P_{i}^{(1)} & =\frac{\gamma B_{p}}{T} \int_{0}^{T} \int_{0}^{z_{\max }} \dot{u}_{i}(t, z) \cos (\omega t) e^{-z / \lambda} d z d t,
\end{aligned}
$$

where $\dot{u}_{i}(t, z)$ is the $i$ th vortex velocity and $T$ is the microwave-field oscillation period.

The number of vortices trapped in the material is estimated by assuming that each vortex carries a single flux quantum $\phi_{0}$ and that vortices are equally distributed over the area $\Sigma$. In turn, the applied magnetic flux during the cool down $B_{0} \Sigma$ is divided into $N_{v}=B_{0} \Sigma / \phi_{0}$ vortices. Thus Eq. (11) can be rewritten as follows:

$$
\begin{aligned}
R_{\mathrm{fl}}= & \frac{2 \mu_{0}^{2} B_{0}}{\phi_{0} B_{p}^{2}} \frac{\sum_{i}^{N_{v}} P_{i}^{(1)}}{N_{v}} \\
& =\frac{2 \mu_{0}^{2} B_{0}}{\phi_{0} B_{p}^{2}}\left\langle P^{(1)}\right\rangle,
\end{aligned}
$$

where $\left\langle P^{(1)}\right\rangle$ represents the average of the dissipated power of $N_{v}$ vortices.

By applying the same definitions used in Eq. (9), we can rewrite the single-vortex average dissipated power as follows:

$$
\begin{aligned}
\left\langle P^{(1)}\right\rangle= & \frac{\phi_{0} B_{p} \lambda f}{\mu_{0} N_{v}} \\
& \times \sum_{i}^{N_{v}} \int_{0}^{1} \int_{0}^{\tilde{z}_{\max }} \dot{\tilde{u}}_{i}(\tilde{t}, \tilde{z}) \cos (2 \pi \tilde{t}) e^{-\tilde{z}} d \tilde{z} d \tilde{t}
\end{aligned}
$$

A Monte Carlo approach is implemented to estimate $\left\langle P^{(1)}\right\rangle$ by considering a statistical sample of 100 vortices. The vortex equation of motion in Eq. (9) is computed for each of the 100 vortices, each time generating a unique random pinning landscape. $P_{i}^{(1)}$ is calculated once the solution to Eq. (9) approaches the steady state. The estimation of $\left\langle P^{(1)}\right\rangle$ is carried out by averaging the outcome of the singlevortex simulations. An example of the calculation for $l=$ $300 \mathrm{~nm}$ and $f_{p \text {,ave }}=200 \mu \mathrm{N} / \mathrm{m}$ is reported in Fig. 4(a). The colored solid lines represent the single-vortex power $P_{i}^{(1)}$
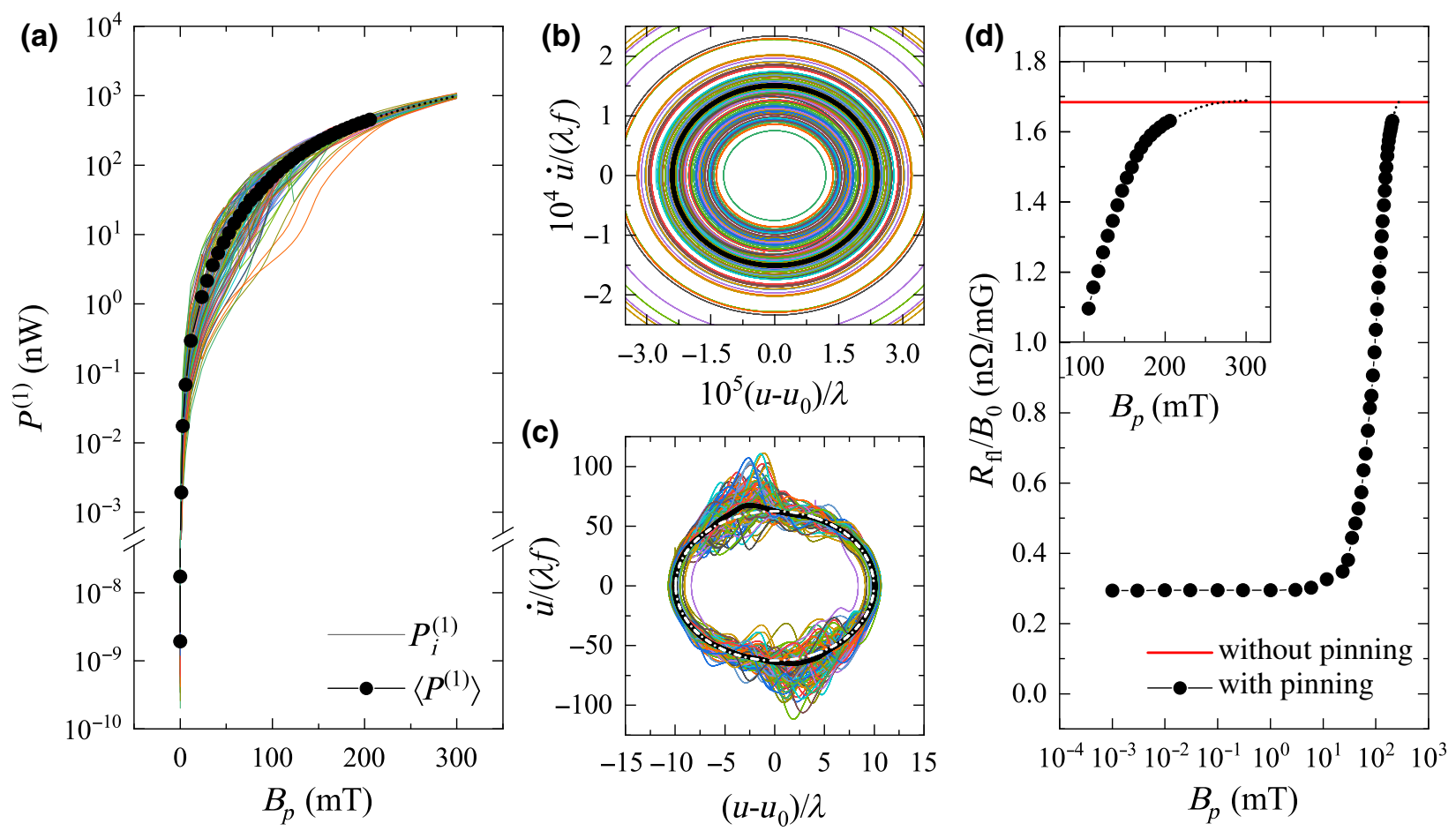

FIG. 4. An example of a vortex-surface-resistance simulation calculated for $l=300 \mathrm{~nm}$ and $f_{p \text {, ave }}=200 \mu \mathrm{N} / \mathrm{m}$ as a function of the peak field. (a) The average single-vortex power dissipation $\left(\left\langle P^{(1)}\right\rangle\right)$, calculated as a function of the peak magnetic field. (b),(c) The average phase portraits calculated over 100 vortex simulations at $0.001 \mathrm{mT}$ and $200 \mathrm{mT}$, respectively. The parameter $u_{0}$ represents the $x$ offset of the oscillation center. The thin colored solid line represents the individual vortex phase portrait, the thick solid black line their average, and the white dash-dotted line the phase portrait in the absence of pinning. (d) The simulated $R_{\mathrm{fl}} / B_{0}$ curve is shown with its abscissa in a semilogarithmic scale. In the inset, the same curve is shown in the range $1 \mathrm{n} \Omega / \mathrm{mG} \leq R_{\mathrm{fl}} / B_{0} \leq 1.8 \mathrm{n} \Omega / \mathrm{mG}$, with its abscissa in a linear scale. In (a) and (d), the dotted line above $200 \mathrm{mT}$ shows how the trend evolves to saturation above the expected maximum $B_{p}$ value that $\mathrm{Nb}$ can sustain, since for the selected pinning landscape, saturation is not achieved below $200 \mathrm{mT}$. 
as a function of $B_{p}$, calculated each time for a random pinning landscape, for a total of 100 vortices, while the solid line with black dots represents the average single-vortex dissipated power $\left\langle P^{(1)}\right\rangle$.

In Figs. 4(b) and 4(c), the average phase portrait of vortex oscillation calculated over 100 vortices, at $B_{p}=$ $0.001 \mathrm{mT}$ and $200 \mathrm{mT}$, respectively, is shown by the thick black solid line. The thin colored lines represent the individual single-vortex phase portraits, while the white dash-dotted line represents the phase portrait of the vortex oscillation in the absence of pinning. Figure 4(d) and its inset show the calculated normalized vortex surface resistance, with the abscissa in semilogarithmic and linear scales, respectively.

At very low microwave fields, the vortex performs oscillations with a small amplitude, since it is well constrained inside a local pinning potential. In this regime, the pinning potential can be approximated by a parabolic function and the vortex equation of motion [Eq. (9)] becomes linear. Thus, the calculated vortex response is linear, implying an elliptical phase portrait and constant surface resistance [Figs. 4(b) and 4(d)].

At high microwave fields, the vortex surface resistance tends to saturate to a constant value equal to the surface resistance in the absence of pinning. Because of the pinning landscape chosen, saturation is not reached for $B_{p}$ values below $200 \mathrm{mT}$ - the theoretical maximum $B_{p}$ value that $\mathrm{Nb}$ can sustain [64]. However, as shown by the simulated values above $200 \mathrm{mT}$ - the dotted line in Fig. 4(d) - the trend of $R_{\mathrm{fl}} / B_{0}$ tends to saturation.

Close to saturation, even if each vortex shows a nonlinear response, the average phase portrait tends to an ellipse [the white dash-dotted line in Fig. 4(c)], meaning that the global response becomes linear for higher microwave fields. Pinning is overcome when the vortices are driven at higher microwave fields and the surface resistance saturates to a constant value equal to the vortex surface resistance in the absence of pinning, as highlighted in the inset of Fig. 4(d).

Such saturation behavior of the vortex surface resistance above a certain microwave field level has also been predicted in Ref. [39] as being generated by viscous losses.In summary, for low microwave fields, pinning can be treated as a parabolic potential - the pinning force is linear and proportional to the vortex displacement - while for high microwave fields, pinning becomes negligible. In both limits, the vortex dynamics are approximated to be linear, leading to a constant surface resistance.

\section{MICROWAVE DEPINNING}

Vortex depinning can explain the nonlinearity of the vortex surface resistance as a function of $B_{p}$ between the two linear limits discussed above. As the microwave field amplitude increases, depinning allows an increasing number of vortices to sample areas with a nonlinear pinning force, leading to the positive slope in the vortex surface resistance. Simulations of single-vortex dynamics allow us to shed light into this mechanism.

In Fig. 5, an example of single-vortex dynamics is reported. The single-vortex power dissipation $\left(P_{i}^{(1)}\right)$ curve shown in Fig. 5(a) is selected from Fig. 4(a). The curve presents a steplike behavior between $63 \mathrm{mT}$ and $75 \mathrm{mT}$ as a function of $B_{p}$. Such an effect is consequence of a "vortex jump," where the vortex gets depinned from a location and captured by a stronger pinning center nearby, as a consequence of microwave depinning.

This behavior is analogous to flux creep [65], for which thermal agitation depins single vortices. Usually in the dc regime, flux creep may initiate flux jumps [66] that quench the superconductive state. In the rf and microwave regimes, depinning and vortex jumps do not generate quenches, since the electromagnetic field "shakes" only a portion of the vortex rather than initiating an avalanchelike displacement of flux lines.

The simulations in Figs. 5(b)-5(d) show the vortexjump behavior just discussed and the microwave depinning phenomenon. The darker blue line in the phase portraits represents steady-state oscillations, while the simulations beneath show the time evolution of the vortex oscillation in the steady state only (for clarity). The simulations are performed for $B_{p}=63 \mathrm{mT}, 75 \mathrm{mT}$, and $170 \mathrm{mT}$ and the red dots in Fig. 5(a) show the corresponding single-vortex dissipated power $\left(P_{i}^{(1)}\right)$ at the respective microwave field amplitude values.

At $B_{p}=63 \mathrm{mT}$, the vortex tip oscillates almost depinned from the local pinning center. As soon as $B_{p}$ is increased to $75 \mathrm{mT}$, the oscillation widens and the vortex starts to interact with a nearby pinning center, which eventually captures it.

Comparing the two simulations, it appears clear that because of the jump from one pinning center to a stronger one, the amplitude of the steady-state oscillations is reduced and so is the oscillation velocity. As a result, the power dissipation is reduced accordingly, generating this abrupt step characteristic in the $B_{p}$ dependence of $P_{i}^{(1)}$.

The progressive microwave depinning phenomenon can be appreciated by comparing the simulations at $75 \mathrm{mT}$ and $170 \mathrm{mT}$. At $B_{p}=75 \mathrm{mT}$, the vortex oscillation is well constrained by the pinning center, while for higher peakmagnetic-field values, the oscillation widens substantially, reaching areas outside the local pinning potential. If the conditions are right, a vortex jump induced by microwave depinning can repeat at higher fields, as shown in Fig. 5(a) around $180 \mathrm{mT}$.

Each vortex may experience jumps and depinning at different $B_{p}$ values depending on the local pinning landscape, producing a unique $P_{i}^{(1)}$ versus $B_{p}$ characteristic. In the 


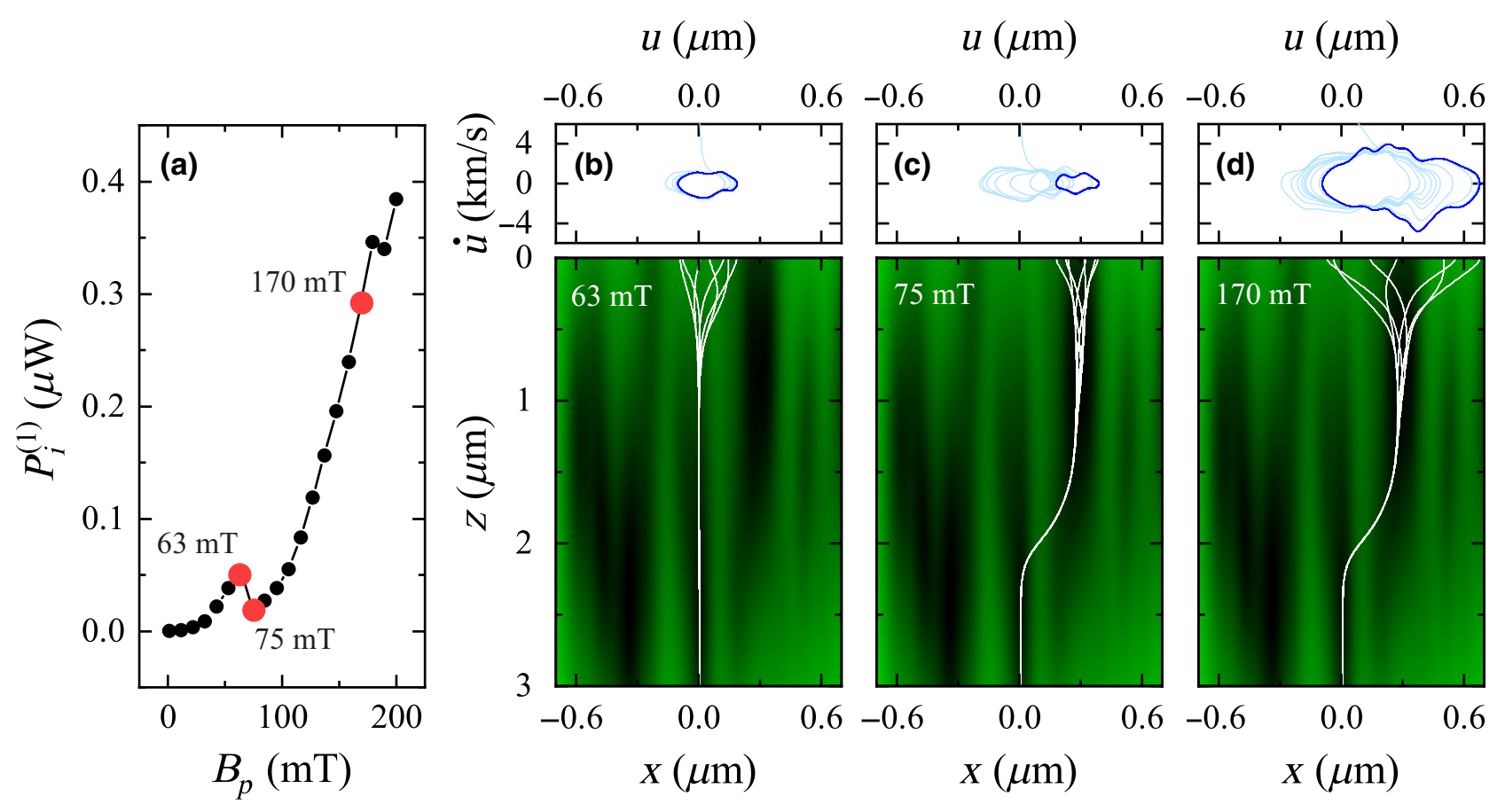

FIG. 5. An example of single-vortex dynamics as a function of the microwave field. (a) The single-vortex power dissipation as a function of the peak magnetic field. (b)-(d) Phase portraits and vortex displacements as a function of the time calculated at the field values $B_{p}=63 \mathrm{mT}, 75 \mathrm{mT}$, and $170 \mathrm{mT}$. In (a), the respective values of the single-vortex dissipated power are highlighted by red dots.

global picture, the microwave-amplitude dependence of $R_{\mathrm{fl}}$ is defined by the collective vortex motion and the abrupt steps expected in the $P_{i}^{(1)}$ due to individual vortex jumps are averaged out.

It is worth mentioning that once a vortex jumps to a new pinning position, it is constrained to that new configuration unless $B_{p}$ is raised to a value high enough to allow for an additional jump. This implies that any vortex experiencing jumps shows hysteretic behavior once $B_{p}$ is decreased back to low amplitudes. However, only a fraction of vortices jump from one pinning center to another (as shown by our simulations), resulting globally in negligible hysteresis on the $R_{\mathrm{fl}}$ trend, as also demonstrated by the microwave measurements.

\section{COMPARISON WITH EXPERIMENTAL DATA}

\section{A. Microwave data}

In this section, we compare the simulation with the available experimental data. Due to the unknown form of the pinning landscape a priori, we compare the data against simulations performed for a collection of pinning landscapes, varying the average pinning force $f_{p \text {,ave }}$, the mean free path $l$, and the dimension of the pinning centers $b \xi$. The simulations are reported in Figs. 6(a)-6(c).

As shown in Fig. 6(a), for a larger average pinning force, the $B_{p}$ value at which saturation is reached increases and the overall $R_{\mathrm{fl}}$ curve is shifted to lower values. The effect of a different mean free path is shown in Fig. 6(b). The flux-flow viscosity decreases for low $l$ values, the vortex moves more freely, and the saturation value increases. The slope of the $R_{\mathrm{fl}}$ microwave-field-amplitude dependence is instead dictated by the dimension of the pinning centers, as shown in Fig. 6(c). The smaller the pinning center, the steeper is the trend and the earlier is saturation reached.

The output of these simulations is solely defined by the choice of the pinning landscape, which is unknown a priori. For this reason, it is virtually impossible to obtain quantitative results. Nevertheless, the simulations can qualitatively describe the experimental data, highlighting that progressive microwave depinning is the likely culprit for the microwave-field dependence of the vortex surface resistance - the higher the driving force, the less constrained is the vortex and the wider are the oscillations, leading to higher resistance.

However, the experimental data do not present saturation at high $B_{p}$ values. This likely occurs because the microwave field level reached before quenching is not high enough to completely depin the vortices and reach saturation. In almost all the simulations, the saturation onset is reached before $200 \mathrm{mT}$ because the pinning landscape considered is a simplification of the real one and is inevitably not accurate enough. In addition, as shown with low-energy $\mu$ SR measurements [67], cavities treated in the same way as the one under study are characterized by a mean free path that varies substantially as a function 

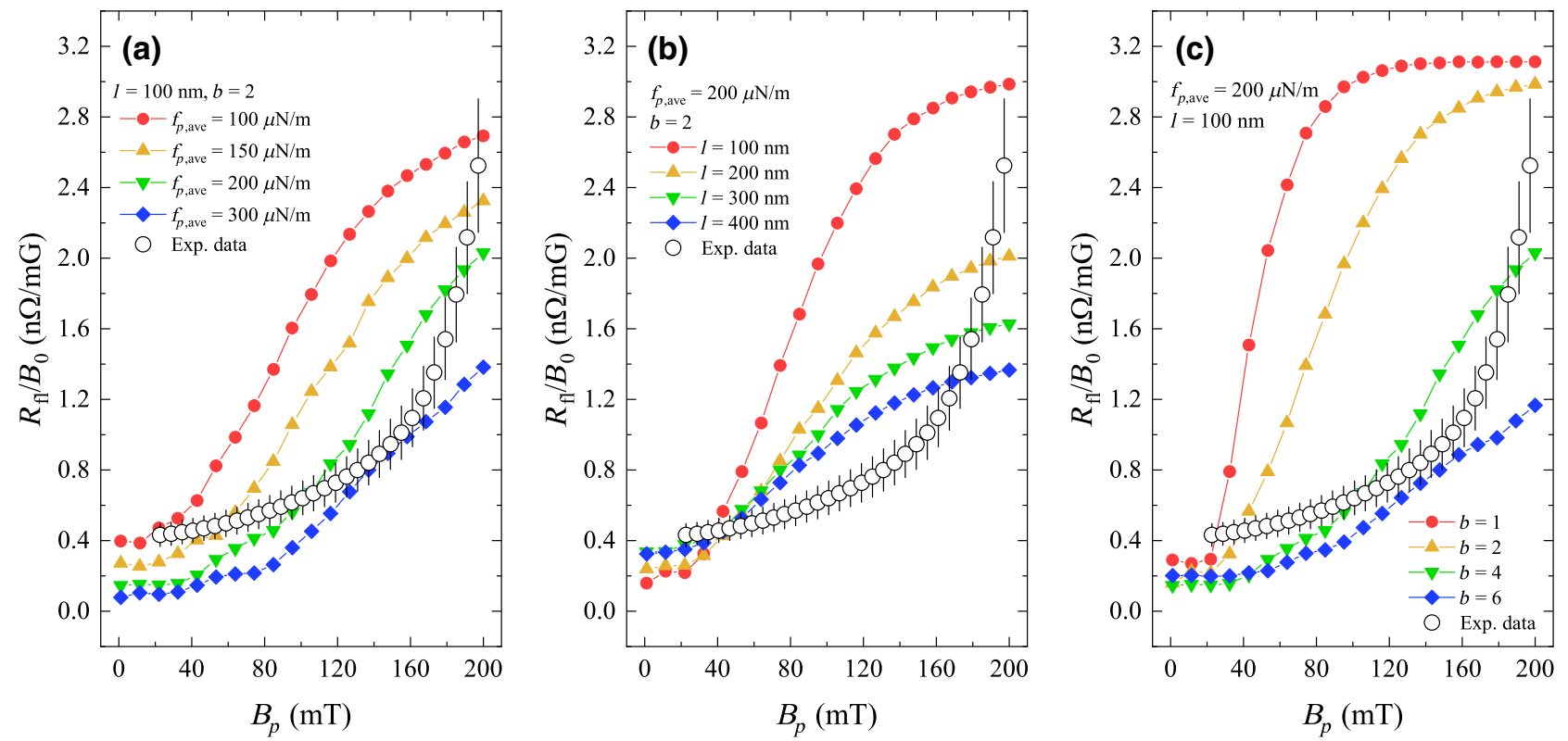

FIG. 6. A comparison of the simulated normalized vortex surface resistance with the experimental data. (a) Simulations for different values of the average pinning force at a fixed mean free path and a fixed factor $b$. (b) Simulations as a function of the mean-free-path value for a fixed average pinning force and factor $b$. (c) Simulations as a function of the factor $b$ for a fixed average pinning force and mean free path.

of the depth, further complicating the problem. Detailed knowledge of the pinning landscape and the mean-freepath profile in the material is necessary in order to correctly interpolate the data with the model presented here.

Other nonlinearities may also play a role in the vortex dynamics. For instance, the vortex flux-flow viscosity might decrease with the velocity, as described by Larkin and Ovchinnikov (LO) [68]. During vortex motion, the energy of quasiparticles in the vortex core increases and when it reaches approximately $\Delta$, the quasiparticles diffuse out of the vortex into the superconductor, the vortex core gets depleted and shrinks in dimension, and the fluxflow viscosity decreases, increasing the dissipation. This tendency is counteracted by the relaxation of the quasiparticles back into the vortex core, where $\tau_{\varepsilon}^{-1}$ is the rate at which this process happens. The shorter $\tau_{\varepsilon}$, the faster the vortex must travel to meet the onset of instability. For extremely dirty $\mathrm{Nb}$, the onset of this nonequilibrium effect is of the order of a couple of $\mathrm{km} / \mathrm{s}$ [69-71], which is close to the values that we simulate at high $B_{p}$. However, no LO data are available for niobium with the levels of impurity that we are investigating. Future dedicated experiments will further explore this possibility.

\section{B. Geometrical considerations}

Due to the nonplanar geometry of the SRF cavity, the vortex surface resistance is not constant across the surface; rather, it varies as described previously in Sec. II. The temperature variation $\Delta T_{\text {out }}$-calculated as the subtraction of the ZFC Tmap data from the FC Tmap data - is directly related to the surface resistance due to the vortices. The surface resistance is directly proportional to the power dissipated, as shown in Eq. (11), which, in turn, is directly proportional to the temperature variation on the outside surface of the cavity through the thermal transport law, i.e., $R_{\mathrm{fl}} \propto \Delta T_{\mathrm{out}} / B_{p}^{2}$.

Figure 7 reports the normalized data shown in Fig. 2(c) averaged over the board number as a function of the angle $\theta$ between $B_{0} \hat{z}$ and the microwave currents flowing parallel to the cavity surface. $\theta$ is estimated as the angle between $B_{0} \hat{z}$ and the cavity surface tangent at each thermometer location.

The error bars are defined to be the standard deviation of the $\Delta T_{\text {out }} / B_{p}^{2}$ average value in the ordinate, while for the values in the abscissa the error is estimated a posteriori to be $10^{\circ}$.

The experimental data are compared against the model presented here, where the Lorentz force $\sin (\theta)$ dependence is taken into consideration, as well as the local surface current distribution at the cavity surface, reported in Fig. 2(b).

The calculation is performed with the same Monte Carlo approach described above, in which every point is calculated averaging the dissipated power over 100 vortices. The simulation is carried out assuming $l=100 \mathrm{~nm}$, $f_{p \text {,ave }}=200 \mu \mathrm{N} / \mathrm{m}$, and $b=4$ but any other set of parameters returns comparable results. In order to compare the experimental data and the simulation, all the quantities in the ordinate are normalized between 0 and 1 . 


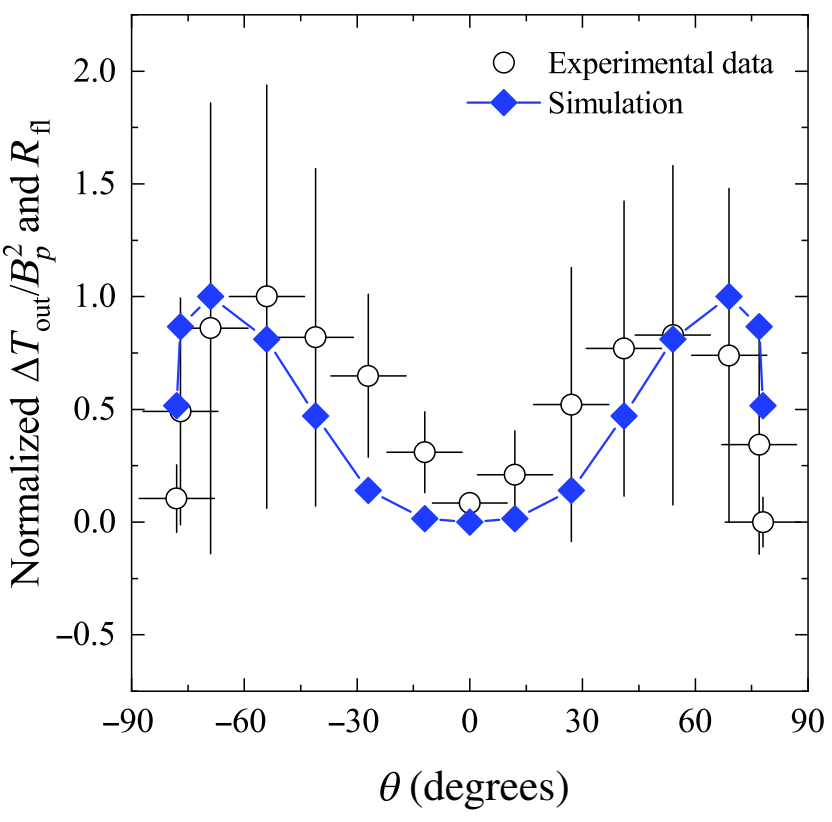

FIG. 7. The normalized experimental data $\Delta T_{\text {out }} / B_{p}^{2}$ and the normalized simulated $R_{\mathrm{fl}}$ plotted against the angle between the microwave currents and the magnetic flux directions.

The angle dependence calculated is in good agreement with the experimental data. At $\theta=0^{\circ}$, the surface resistance is zero, since the trapped field is parallel to the microwave currents, while at $\theta \simeq 80^{\circ}$, the surface resistance decreases because the microwave current drops rapidly to zero approaching the cavity irises.

\section{CONCLUSIONS}

In this work, the vortex surface resistance as a function of the microwave field amplitude is simulated by means of a Monte Carlo approach aimed to solve the equation of motion for a collection of vortices each oscillating within a randomly defined pinning landscape.

Simulations suggest that at low microwave amplitudes, the vortices are well constrained within a local pinning potential and their response is linear, leading to a constant surface resistance. For increasing field amplitudes, the vortices may oscillate out of their local pinning potential and possibly jump from their local pinning center to a neighboring one, introducing nonlinearity. The effect of such phenomena leads to an increase of the vortex surface resistance until the average response approaches a linear behavior for higher field amplitudes, where the average vortex motion becomes insensitive to the pinning landscape. When this happens, the surface resistance saturates to a constant value.

The slope, saturation value, and saturation onset are closely related to the pinning landscape - not known $a$ priori - within which the vortices oscillate. A quantitative description of $R_{\mathrm{fl}}$ as a function of $B_{p}$ is then virtually impossible. Nevertheless, the experimental data are in qualitative agreement with the simulations.

The experimental data as a function of the microwave field amplitude do not show saturation for $B_{p}$ values lower than the quenching field. As described above, this might be due an insufficiently accurate choice of the pinning parameters and of the mean-free-path value in the simulations or to the onset of other nonlinearities such as LO instability at high fields.

To conclude, this work allows us to gain insights into the vortex dynamics under a high-amplitude microwave drive and on the transition from low to high field amplitudes. The microscopic nature of the microwave-field dependence of the vortex surface resistance is presented and attributed to microwave depinning of vortices. Appreciable contributions from thermal-feedback effects for the magnetic field values and microwave amplitudes explored experimentally $-B_{0}<100 \mathrm{mG}$ and $B_{p}<200 \mathrm{mT}$-were, on the other hand, ruled out.

\section{ACKNOWLEDGMENTS}

We thank Martina Martinello and Alex Romanenko for insightful discussions that helped the development of this work. This work was supported by the U.S. Department of Energy, Office of High Energy Physics. Fermilab is operated by Fermi Research Alliance, LLC under Contract No. DE-AC02-07CH11359 with the U.S. Department of Energy.

[1] A. A. Abrikosov, On the magnetic properties of superconductors of the second group, Zh. Eksp. Teor. Fiz. 32, 1442 (1957); A. A. Abrikosov, Soviet Phys. JETP 5, 1174 (1957).

[2] L. V. Shubnikov, V. I. Khotkevich, Yu. D. Shepelev, and Yu. N. Ryabinin, Magnetic properties of superconducting metals and alloys, Zh. Eksp. Teor. Fiz. 7, 221 (1937); L. V. Shubnikov, V. I. Khotkevich, Yu. D. Shepelev, and Yu. N. Ryabinin, Ukr. J. Phys. 57, 42 (2008).

[3] A. Romanenko, A. Grassellino, O. Melnychuk, and D. A. Sergatskov, Dependence of the residual surface resistance of superconducting radio frequency cavities on the cooling dynamics around $T_{c}$, J. Appl. Phys. 115, 184903 (2014).

[4] A. Romanenko, A. Grassellino, A. C. Crawford, D. A. Sergatskov, and O. Melnychuk, Ultra-high quality factors in superconducting niobium cavities in ambient magnetic fields up to $190 \mathrm{mG}$, Appl. Phys. Lett. 105, 234103 (2014).

[5] S. Posen, M. Checchin, A. C. Crawford, A. Grassellino, M. Martinello, O. S. Melnychuk, A. Romanenko, D. A. Sergatskov, and Y. Trenikhina, Efficient expulsion of magnetic flux in superconducting radiofrequency cavities for high $Q_{0}$ applications, J. Appl. Phys 119, 213903 (2016).

[6] M. Martinello, A. Grassellino, M. Checchin, A. Romanenko, O. Melnychuk, D. A. Sergatskov, S. Posen, and J. F. Zasadzinski, Effect of interstitial impurities on the field dependent microwave surface resistance of niobium, Appl. Phys. Lett. 109, 062601 (2016). 
[7] D. C. Mattis and J. Bardeen, Theory of the anomalous skin effect in normal and superconducting metals, Phys. Rev. 111, 412 (1958).

[8] A. Gurevich, Theory of rf superconductivity for resonant cavities, Supercond. Sci. Technol. 30, 034004 (2017).

[9] T. Kubo and A. Gurevich, Field-dependent nonlinear surface resistance and its optimization by surface nanostructuring in superconductors, Phys. Rev. B 100, 064522 (2019).

[10] A. Romanenko, F. Barkov, L. D. Cooley, and A. Grassellino, Proximity breakdown of hydrides in superconducting niobium cavities, Supercond. Sci. Technol. 26, 035003 (2013).

[11] A. Romanenko and D. I. Schuster, Understanding Quality Factor Degradation in Superconducting Niobium Cavities at Low Microwave Field Amplitudes, Phys. Rev. Lett. 119, 264801 (2017).

[12] A. Romanenko, R. Pilipenko, S. Zorzetti, D. Frolov, M. Awida, S. Belomestnykh, S. Posen, and A. Grassellino, Three-Dimensional Superconducting Resonators at $t<$ $20 \mathrm{mK}$ with Photon Lifetimes up to $\tau=2 \mathrm{~s}$, Phys. Rev. Appl. 13, 034032 (2020).

[13] M. Cardona, G. Fischer, and B. Rosenblum, Microwave Surface Impedance of Superconductors of the Second Kind: In-Bi Alloys, Phys. Rev. Lett. 12, 101 (1970).

[14] J. I. Gittleman and B. Rosenblum, Radio-Frequency Resistance in the Mixed State for Subcritical Currents, Phys. Rev. Lett. 16, 734 (1966).

[15] M. Rabinowitz, Analysis of a critical loss in a superconductor, J. Appl. Phys. 42, 88 (1971).

[16] H. R. Segal and W. L. McLean, Magnetic field dependence of the microwave surface resistance of pure niobium, J. Low Temp. Phys. 22, 141 (1976).

[17] C. C. Chin, D. E. Oates, G. Dresselhaus, and M. S. Dresselhaus, Nonlinear electrodynamics of superconducting $\mathrm{NbN}$ and $\mathrm{Nb}$ thin films at microwave frequencies, Phys. Rev. B 45, 4788 (1992).

[18] D. Janjušević, M. S. Grbić, M. Požek, A. Dulčić, D. Paar, B. Nebendahl, and T. Wagner, Microwave response of thin niobium films under perpendicular static magnetic fields, Phys. Rev. B 74, 104501 (2006).

[19] A. Alimenti, N. Pompeo, K. Torokhtii, T. Spina, R. Flükiger, L. Muzzi, and E. Silva, Surface impedance measurements on $\mathrm{Nb}_{3} \mathrm{Sn}$ in high magnetic fields, IEEE Trans. Appl. Supercond. 29, 3500104 (2019).

[20] S. Revenaz, D. Labbé-Lavigne, D. E. Oates, G. Dresselhaus, and M. S. Dresselhaus, Frequency dependence of the surface impedance of $\mathrm{YBa}_{2} \mathrm{Cu}_{3} \mathrm{O}_{7-\delta}$ thin films in a dc magnetic field: Investigation of vortex dynamics, Phys. Rev. B 50, 1178 (1994).

[21] Y. Matsuda, M. B. Gaifullin, K. Kumagai, K. Kadowaki, and T. Mochiku, Collective Josephson Plasma Resonance in the Vortex State of $\mathrm{Bi}_{2} \mathrm{Sr}_{2} \mathrm{CaCu}_{2} \mathrm{O}_{8+\delta}$, Phys. Rev. Lett. 75, 4512 (1995).

[22] M. Golosovsky, M. Tsindlekht, and D. Davidov, Highfrequency vortex dynamics in $\mathrm{YBa}_{2} \mathrm{Cu}_{3} \mathrm{O}_{7}$, Supercond. Sci. Technol. 9, 1 (1996).

[23] K. Hashimoto, T. Shibauchi, T. Kato, K. Ikada, R. Okazaki, H. Shishido, M. Ishikado, H. Kito, A. Iyo, H. Eisaki, S. Shamoto, and Y. Matsuda, Microwave Penetration Depth and Quasiparticle Conductivity of $\mathrm{PrFeAsO}_{1-y}$ Single
Crystals: Evidence for a Full-Gap Superconductor, Phys. Rev. Lett. 102, 017002 (2009).

[24] K. Hashimoto, T. Shibauchi, S. Kasahara, K. Ikada, S. Tonegawa, T. Kato, R. Okazaki, C. J. van der Beek, M. Konczykowski, H. Takeya, K. Hirata, T. Terashima, and Y. Matsuda, Microwave Surface-Impedance Measurements of the Magnetic Penetration Depth in Single Crystal $\mathrm{Ba}_{1-x} \mathrm{~K}_{x} \mathrm{Fe}_{2} \mathrm{As}_{2}$ Superconductors: Evidence for a Disorder-Dependent Superfluid Density, Phys. Rev. Lett. 102, 207001 (2009).

[25] T. Okada, H. Takahashi, Y. Imai, K. Kitagawa, K. Matsubayashi, Y. Uwatoko, and A. Maeda, Microwave surfaceimpedance measurements of the electronic state and dissipation of magnetic vortices in superconducting LiFeAs single crystals, Phys. Rev. B 86, 064516 (2012).

[26] M. W. Coffey and J. R. Clem, Vortex-Motion Dissipation in High- $T_{c}$ Superconductors at Microwave Frequencies, Phys. Rev. Lett. 67, 386 (1991).

[27] R. Marcon, R. Fastampa, M. Giura, and E. Silva, Vortex-motion dissipation in high- $T_{c}$ superconductors at microwave frequencies, Phys. Rev. B 43, 2940 (1991).

[28] E. B. Sonin, A. K. Tagantsev, and K. B. Traito, Two-mode electrodynamics of superconductors in the mixed state, Phys. Rev. B 43, 5830 (1992).

[29] A. Grassellino, A. Romanenko, D. A. Sergatskov, O. Melnychuk, Y. Trenikhina, A. C. Crawford, A. Rowe, M. Wong, T. Khabiboulline, and F. Barkov, Nitrogen and argon doping of niobium for superconducting radio frequency cavities: A pathway to highly efficient accelerating structures, Supercond. Sci. Tech. 26, 102001 (2013).

[30] M. Martinello, M. Checchin, A. Romanenko, A. Grassellino, S. Aderhold, S. K. Chandrasekeran, O. Melnychuk, S. Posen, and D. A. Sergatskov, Field-Enhanced Superconductivity in High-Frequency Niobium Accelerating Cavities, Phys. Rev. Lett. 121, 224801 (2018).

[31] B. Piosczyk, P. Kneisel, O. Stoltz, and J. Halbritter, Investigations of additional losses in superconducting niobium cavities due to frozen-in flux, IEEE Trans. Nucl. Sci. 20, 108 (1973).

[32] C. Benvenuti, S. Calatroni, I. E. Campisi, P. Darriulat, M. A. Peck, R. Russo, and A.-M. Valente, Study of the surface resistance of superconducting niobium films at 1.5 GHz, Physica C 316, 153 (1999).

[33] A. Gurevich and G. Ciovati, Effect of vortex hotspots on the radio-frequency surface resistance of superconductors, Phys. Rev. B 87, 054502 (2013).

[34] D. Gonnella, J. Kaufman, and M. Liepe, Impact of nitrogen doping of niobium superconducting cavities on the sensitivity of surface resistance to trapped magnetic flux, J. Appl. Phys 119, 073904 (2016).

[35] M. Checchin, M. Martinello, A. Grassellino, A. Romanenko, and J. F. Zasadzinski, Electron mean free path dependence of th vortex surface impedance, Supercond. Sci. Technol. 30, 034003 (2017).

[36] M. Checchin, M. Martinello, A. Grassellino, S. Aderhold, S. K. Chandrasekaran, O. S. Melnychuk, S. Posen, A. Romanenko, and D. A. Sergatskov, Frequency dependence of trapped flux sensitivity in SRF cavities, Appl. Phys. Lett. 112, 072601 (2018).

[37] S. Calatroni and R. Vaglio, Surface resistance of superconductors in the presence of a dc magnetic field: Frequency 
and field intensity limits, IEEE Trans. Appl. Supercond. 27, 3500506 (2017).

[38] S. Calatroni and R. Vaglio, Simple model for the rf field amplitude dependence of the trapped flux sensitivity in superconducting rf cavities, Phys. Rev. Accel. Beams 22, 022001 (2019).

[39] D. B. Liarte, D. Hall, P. N. Koufalis, A. Miyazaki, A. Senanian, M. Liepe, and J. P. Sethna, Vortex Dynamics and Losses due to Pinning: Dissipation from Trapped Magnetic Flux in Resonant Superconducting Radio-Frequency Cavities, Phys. Rev. Appl. 10, 054057 (2018).

[40] B. Aune, Superconducting TESLA cavities, Phys. Rev. ST Accel. Beams 3, 092001 (2000).

[41] J. Knobloch, H. Muller, and H. Padamsee, Design of a high speed, high resolution thermometry system for $1.5 \mathrm{GHz}$ superconducting radio frequency cavities, Rev. Sci. Instrum. 65, 3521 (1994).

[42] O. Melnychuk, A. Grassellino, and A. Romanenko, Error analysis for intrinsic quality factor measurement in superconducting radio frequency resonators, Rev. Sci. Instrum. 85, 124705 (2014).

[43] F. London and H. London, The electromagnetic equations of the supraconductors, Proc. R. Soc. Lond. A 149, 71 (1935).

[44] E. H. Brandt, The flux-line lattice in superconductors, Rep. Prog. Phys. 58, 1465 (1995).

[45] Differently than our previous approach in which $B_{p} \rightarrow 0$ $[35,36]$, in this work we consider the line tension to describe the vortex dynamics in more general way. The microwave excitation has a large amplitude and due to the finite line tension, the vortexes oscillate much more deeply than $\lambda$.

[46] J. Bardeen and M. J. Stephen, Theory on the motion of vortices in superconductors, Phys. Rev. 140, A1197 (1965).

[47] M. Tinkham, Introduction to Superconductivity (Dover Publication, Inc., Mineola, New York, 2004).

[48] A. B. Pippard, An experimental and theoretical study of the relation between magnetic field and current in a superconductor, Proc. R. Soc. Lond. A 216, 547 (1953).

[49] C. J. Gorter and H. Casimir, On supraconductivity I, Physica 1, 306 (1934).

[50] J. Bardeen, L. N. Cooper, and J. R. Schrieffer, Theory of superconductivity, Phys. Rev. 108, 1175 (1957).

[51] A. Suter, E. Morenzoni, N. Garifianov, R. Khasanov, E. Kirk, H. Luetkens, T. Prokscha, and M. Horisberger, Observation of nonexponential magnetic penetration profiles in the Meissner state: A manifestation of nonlocal effects in superconductors, Phys. Rev. B 72, 024506 (2005).

[52] T. P. Sheehan, Rules for the energy gap and critical field of superconductors, Phys. Rev. 149, 368 (1966).

[53] B. W. Maxfield and W. L. McLean, Superconducting penetration depth of niobium, Phys. Rev. 139, A1515 (1965).

[54] V. L. Ginzburg and L. D. Landau, On the theory of superconductivity, Zh. Eksp. Teor. Fiz. 20, 1064 (1950); L. D. Landau, Collected Papers (Pergamon Press, New York, 1965).

[55] M. Martinello, in 19th International Conference on $R F$ Superconductivity, Dresden, Germany, 2019, (to be published).
[56] C. Z. Antoine, Influence of crystalline structure on rf dissipation in superconducting niobium, Phys. Rev. Accel. Beams 22, 034801 (2019).

[57] Y. Trenikhina, A. Romanenko, J. Kwon, J.-M. Zuo, and J. F. Zasadzinski, Nanostructural features degrading the performance of superconducting radio frequency niobium cavities revealed by transmission electron microscopy and electron energy loss spectroscopy, J. Appl. Phys. 117, 154507 (2015).

[58] E. V. Thuneberg, J. Kurkijärvi, and D. Rainer, Elementaryflux-pinning potential in type-II superconductors, Phys. Rev. B 29, 3913 (1984).

[59] L. Embon, Y. Anahory, A. Suhov, D. Halbertal, J. Cuppens, A. Yakovenko, A. Uri, Y. Myasoedov, M. L. Rappaport, M. E. Huber, A. Gurevich, and E. Zeldov, Probing dynamics and pinning of single vortices in superconductors at nanometer scales, Sci. Rep. 5, 7598 (2015).

[60] R. Labusch, Calculation of the critical field gradient in typeII superconductors, Cryst. Lattice Defects 1, 1 (1969).

[61] A. S. Dhavale, P. Dhakal, A. A. Polyanskii, and G. Ciovati, Flux pinning characteristics in cylindrical niobium samples used for superconducting radio frequency cavity fabrication, Supercond. Sci. Technol. 25, 065014 (2012).

[62] L. H. Allen and J. H. Claassen, Technique for measuring the elementary pinning force in thin films, Phys. Rev. B 39, 2054 (1989).

[63] G. S. Park, C. E. Cunningham, B. Cabrera, and M. E. Huber, Vortex Pinning Force in a Superconducting Niobium Strip, Phys. Rev. Lett. 68, 1920 (1992).

[64] S. Posen, N. Valles, and M. Liepe, Radio Frequency Magnetic Field Limits of $\mathrm{Nb}$ and $\mathrm{Nb}_{3} \mathrm{Sn}$, Phys. Rev. Lett. 115, 047001 (2015).

[65] P. W. Anderson, Theory of Flux Creep in Hard Superconductors, Phys. Rev. Lett. 9, 309 (1962).

[66] R. Prozorov, D. V. Shantsev, and R. G. Mints, Collapse of the critical state in superconducting niobium, Phys. Rev. B 74, 220511(R) (2006).

[67] A. Romanenko, A. Grassellino, F. Barkov, A. Suter, Z. Salman, and T. Prokscha, Strong Meissner screening change in superconducting radio frequency cavities due to mild baking, Appl. Phys. Lett. 104, 072601 (2014).

[68] A. I. Larkin and Yu. N. Ovchinnikov, Nonlinear conductivity of superconductors in the mixed state, Zh. Eksp. Teor. Fiz. 68, 1915 (1975); A. I. Larkin and Yu. N. Ovchinnikov, Sov. Phys.-JETP, 41, 690 (1976).

[69] G. Grimaldi, A. Leo, D. Zola, A. Nigro, S. Pace, F. Laviano, and E. Mezzetti, Evidence for low-field crossover in the vortex critical velocity of type-II superconducting thin films, Phys. Rev. B 82, 024512 (2010).

[70] A. Leo, G. Grimaldi, R. Citro, A. Nigro, S. Pace, and R. P. Hubener, Quasiparticle scattering time in niobium superconducting films, Phys. Rev. B 84, 014536 (2011).

[71] O. V. Dobrovolskiy, V. A. Shklovskij, M. Hanefeld, M. Zörb, L. Köhs, and M. Huth, Pinning effects on flux flow instability in epitaxial $\mathrm{Nb}$ thin films, Supercond. Sci. Technol. 30, 085002 (2017). 